\title{
Research of Mobile Augmented Reality Technology Applied in Agriculture
}

\author{
Ming Liu' ${ }^{1}$ Xiao Li ${ }^{1}$, Xiao Lei ${ }^{1}$, Sheng Wu ${ }^{2}$ \\ ${ }^{1}$ Department of Computer and Information Science, Southwest University, Chongqing, China \\ ${ }^{2}$ National Engineering Research Center for Information Technology in Agriculture, Beijing, China \\ liuming0305@gmail.com, li.xiao.uu@gmail.com
}

\begin{abstract}
The application of mobile augmented reality in agriculture can effectively promote the digital agriculture and virtual agriculture, which is helpful to accelerate the transformation of agricultural science and technology achievements. This paper focuses on the design of technical framework of mobile augmented reality for agriculture, which includes physical layer network layer, decision layer and application layer. At the same time, it analyses the key technology of mobile augmented reality applications in agriculture system such as tracking technology, human-computer interaction technology and data storage and access technology. Also, the author researches the application of mobile augmented reality technology in agriculture and discusses the development of it in the field of agriculture.

Index Terms - Mobile augmented reality, agriculture, technical framework, key technology.
\end{abstract}

\section{Introduction}

In recent years, digital agriculture [1] and virtual agriculture [2] greatly promote the development of agriculture in our country. In agricultural production and display, mobile augmented reality [3] technology is applied more widely. Mobile augmented reality technology mainly refers to the augmented reality [4] technology on mobile terminal, such as handheld computer, intelligent mobile phone, portable computer and other equipment. It combines computer graphics technology, multimedia technology, sensor technology, human-computer interaction technology, stereo display technology and simulation technology. Its main task is in the mobile platform of virtual objects and real environment realtime fusion, and it has the characteristics of fusion of virtual and reality, real-time interaction, 3D registration.

In agricultural production, it is very important to increasing agricultural production efficiency that we can enhance the growth of crops in the process of information acquisition capability and field management level. MAR can be more intuitive to reflect the growth status of crops, assisting the user to make a decision. In addition, in the tourism agriculture, popularizing knowledge of agricultural with MAR is novel and entertaining, which is popular to tourists. Therefore, for the research of mobile augmented reality technology application in agriculture, the design of mobile augmented reality application of agricultural technology architecture has important theoretical significance and practical value.

\section{Technology Framework of MAR Agricultural Application}

Compared to the desktop computer, the mobile devices have small memory, limited computing capability, easy to carry features, while the servers have powerful computing and storage capacity. Using client / server system architecture can make full use of advantages of both hardware environments, to allocate tasks reasonably, and it can effectively reduce the system overhead. Thus, we can apply MAR to the application of agricultural technology architecture into four layers: physical layer, network layer, decision layer and application layer. MAR application in agriculture technology architecture is shown in figure 1 .

\section{A. Physical Layer}

The physical layer is the underlying technology architecture, also the base layer, which determines the communication interface and characteristics of the whole architecture. Its main function is using mobile devices or sensors to collect crops or agricultural information. The mobile terminals include smart phones, PADs, portable computers. The main task is to obtain crop image, transfer and display of data information, also realize the field orientation for some devices with GPS module. Ref. [5] used sensors measure soil moisture and temperature, ambient temperature, relative humidity and light intensity information to calculate irrigation data.

Sensors in MAR agriculture application system generally refer to the temperature sensors, humidity sensors, wind sensors and so on. The sensors in the soil or on the plants collects soil moisture content, conductivity or the air humidity, temperature and other information, Also can provide crop realtime image information, providing data support for augmented reality scene.

\section{B. Network Layer}

Network layer is mainly responsible for information communication of the system, which determines technology selection of system communication. Currently, the common wireless communication mode includes: GPRS, 3G, Wi-Fi, microwave communication, satellite communication etc. With the limitation of the scale of data and the communication speed, $3 \mathrm{G}$ can fulfill the system needs. Ref. [6] used the $3 \mathrm{G}$ mobile phone to transmit data to the administrator system and alarm system, regulating and controlling greenhouse crop growth environment. 


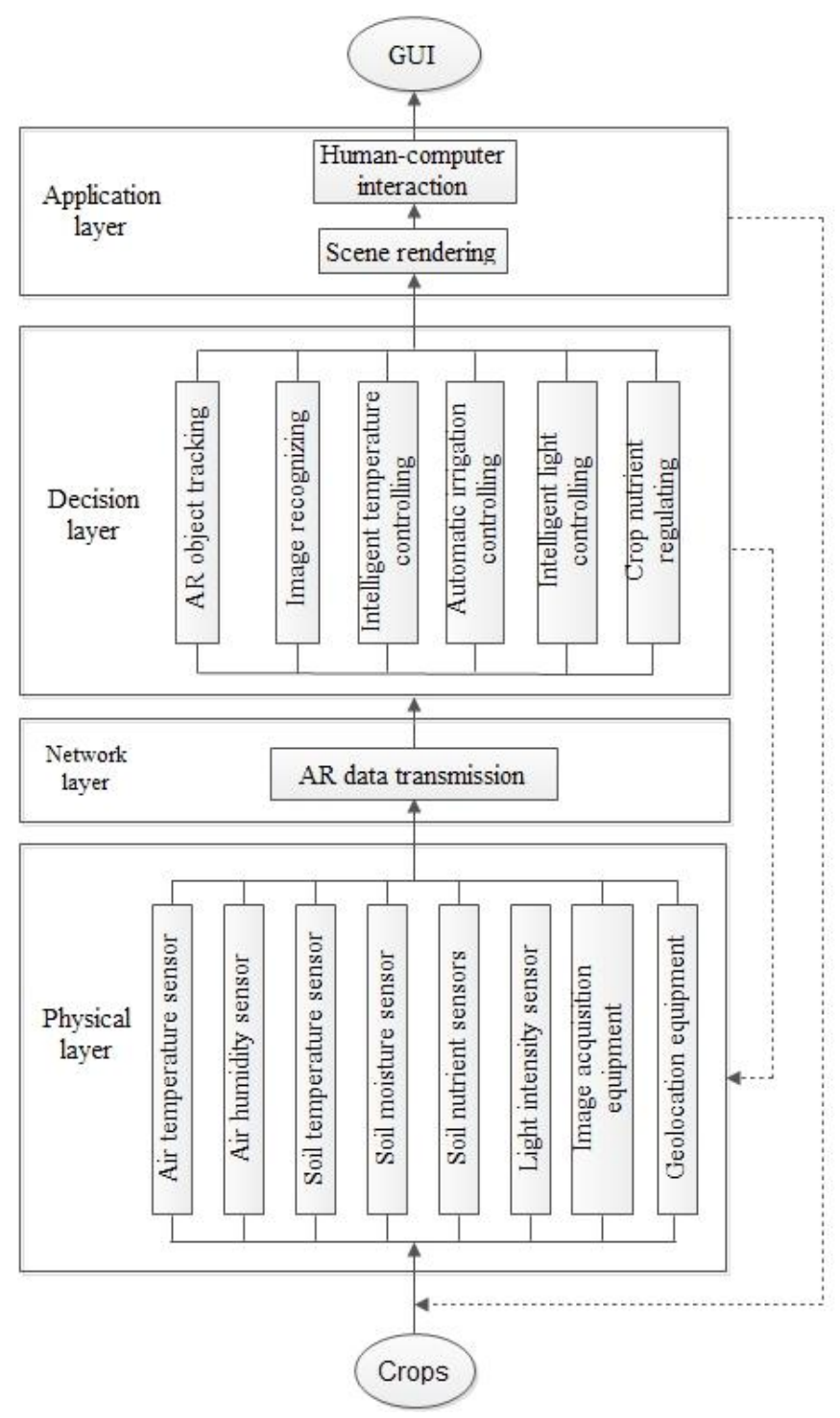

Fig. 1 MAR agricultural application architecture diagram

\section{Decision Layer}

The main task of decision layer is analyzing network data and deciding how to implement the plan. The server uses the PC to connect mobile terminal equipment and Internet, used for data management and analysis, data sharing, integrated advanced decision, user management. It receives the image information from the network layer to identify image crops or agricultural products, and then matches in the database and the preset image, at the same time, real-time tracking based on real scene information collected by physical layer.

\section{Application Layer}

The application layer is at the top of MAR application in agriculture technology architecture, also is the core layer. It determines finally the user experience effect. For example, for a leaf with lesion, the system could give the insecticidal or spraying animation information to the user based on rendering scenes.

\section{III . Key Technology}

\section{A. Tracking Technique}

Tracking technology [3] is the core part for mobile augmented reality technology in agricultural applications, which directly determines whether the virtual information can be accurately superimposed to the real environment. Tracking technique in MAR application in agriculture system is mainly based on natural feature tracking technique. In natural feature tracking system, recognition of feature points is relatively difficult, especially in the crop identification, which makes computational difficulty greatly increased.

\section{B. Human-computer Interaction Techniques}

Mobile augmented reality technology is also a kind of human-computer interaction technology [8]. Because the mobile screens are small without keyboard and have low processing ability, Human-computer interaction techniques in MAR application in agriculture system include:

\section{(1) Touch Interaction}

Touch interactive is a natural, friendly man-machine interactive mode, which allows the user to interact with the system though touching many points with a variety of ways. On mobile platform, interactive mode includes: click, doubleclick, press, rotation, drag etc. In some AR applications, the user can control virtual objects position in the real environment with moving mobile.

\section{(2) Sensor Interaction}

Here the sensor interaction refers to the interaction with mobile devices using the gravity sensor, acceleration sensor, magnetic sensor and gyroscope etc. For example in the maze game, gyroscope and acceleration sensor control the globule to move with detecting the change of tilt angle and speed. There are other advanced interactive modes in MAR system, such as voice interaction.

\section{(3) Data Storage and Access}

Due to the particularity of mobile devices, storage and access data of MAR application in agriculture system is the key problem to be solved. If the system needs to provide the current user associated with the environmental information, the data must conform to the user's current context. By the database and the context based service technology, we can effectively solve the problem of data and service discovery, management, access and other issues.

\section{IV . Application of MAR Technology in Agriculture}

MAR technology could be applied in many agriculture fields, such as growth simulation of virtual animals and plants and virtual breeding. With discussion above, figure 2 show us the components of MAR application in agriculture system.

The system of MAR application in agriculture includes: mobile terminals, sensors, server and network equipment. Mobile cameras acquire crop images information, at the same time, crops information is roughly acquired by GPS. The compass will acquire the user`s orientation, while the gravity sensor and the acceleration sensor acquire inclined angle to 
determinate the plant information and complete crops detecting preliminarily. The plant images are sent to the server for matching, and then some crops information is sent to mobile terminal. According to data such as temperature from sensors, mobile terminal screen display a temperature with a visual thermometer. Combined with other data and real scenes, augmented reality display and interaction are given to us. For example, through analyzing the quantitative and shape of leaves, combined with the seasons, temperature, fertilizer and other information, the system can provide the corresponding suggestion for administrators, achieving efficient management of farmland. Figure 3 show us flow chart of MAR application in agriculture.

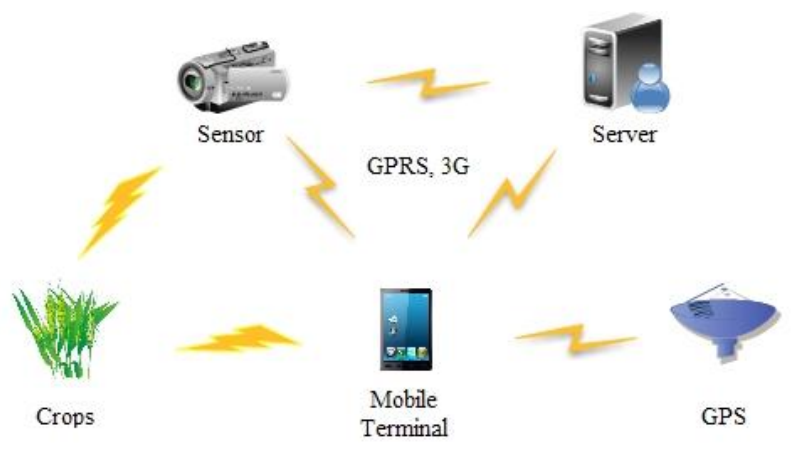

Fig. 2 The components of MAR application in agriculture system

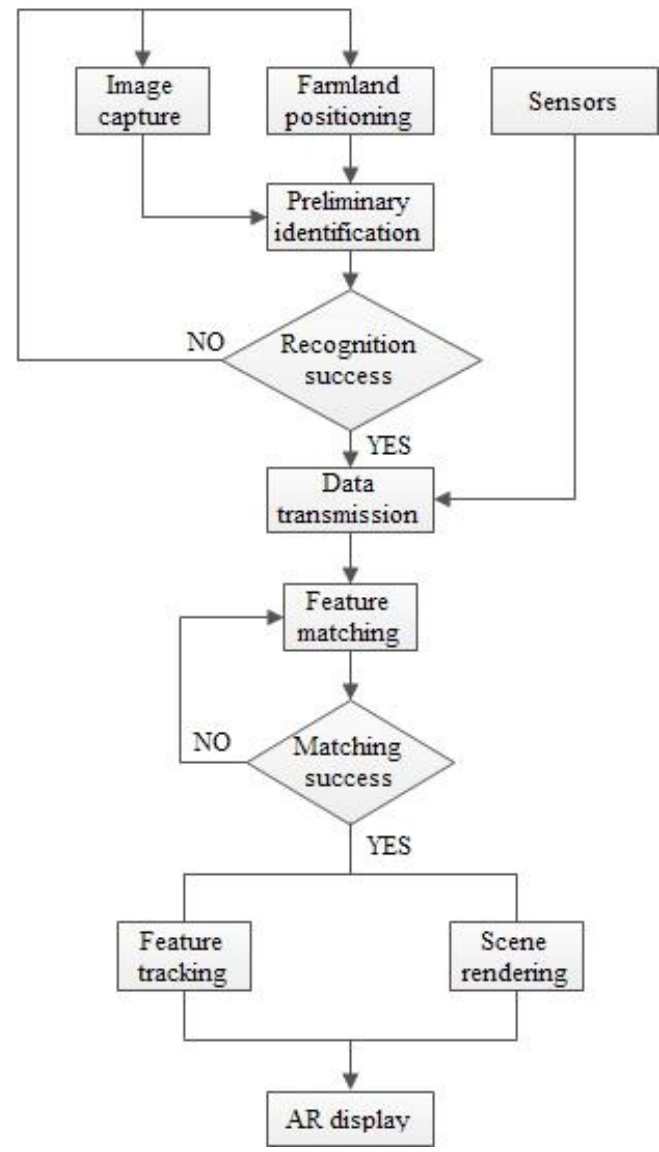

Fig. 3 The flow chart of MAR application in agriculture

\section{V . Conclusions and Future Work}

The paper studies the application of mobile augmented reality technology in agriculture, focusing on the design of MAR application in agriculture technology architecture. And we analyzed key technology of MAR application in agriculture system, which could promote the application digital agriculture and virtual agriculture, accelerating the transformation of agricultural science and technology achievements. On one hand, mobile augmented reality technology itself is still in the initial period of development, so there are still many technical difficulties. It is worth further study. On the other hand, mobile augmented reality technology is novel, visual and entertaining, which makes the technology in the field of agriculture have great research value and practical value. To effectively solve the technical difficulties at present and combine organically with the new methods and other advanced technology, used in agriculture, is the effective way to promote the development of the technology.

\section{VI . Acknowledgment}

The authors thank International Conference on Advanced Computer Science and Electronics Information and reviewers' comments.

\section{References}

[1] Peng Peng, Binggeng Xie, Yinlin Hou, et al, "On the digital agriculture," Research of Agricultural Modernization, vol. 21(7), pp. 254-256, 2000. (In Chinese)

[2] Guocai Yang, "Research of virtual agriculture architecture," Computer Science, vol. 32(3), pp. 125-126. 2005. (In Chinese)

[3] Tobias H. Hollerer, Steven K. Feiner. "Mobile Augmented Reality," Telegeoinformatics: Location-Based Computing and Services, 2004.

[4] Ronald T. Azuma. "A Survey of Augmented Reality," Teleoperators and Virtual Environments, vol. 6(4), pp. 355-385, 1997.

[5] Zhenxia Wen, Long He, Haiqing Yang, et al, "Developing automatic control of precision irrigation technology, accelerating the construction of resource-saving agriculture," Agricultural Equipment \& Technology, vol. 36(3), pp. 22-24, 2010. (In Chinese)

[6] Junfang Ren, Yi Cheng, Xin`e Tao, "Application of 3G technology in agricultural greenhouse in remote control," science and technology world, vol. 18, 2012. (In Chinese)

[7] Zhipeng Zhong, "Research on Application of mobile augmented reality museum guide", Beijing Institute of Technology, 2011. (In Chinese)

[8] Hao Yan, "Research and application of augmented reality system of human-computer interaction technology," Qingdao University, 2011. (In Chinese)

[9] Craig Villamor, Dan Willis, and Luke Wroblewski, "Touch gesture reference guide," http://www.lukew.com/ff/entry.asp?1071, 2010.

[10] Qing Liu, Xiangjian Lian, "Context-Based Dynamic Web Service Composition," Microelectronics and computer, vol. 25(9), pp. 50-52. (In Chinese)

[11] George Papagiannakis, Gurminder Singh, Nadia Magnenat - Thalmann. A survey of mobile and wireless technologies for augmented reality systems. Switzerland, California.2008.

[12] Behrang Parhizkar, Ashraf Abbas M. AL - Modwahi, Arash HabibiLashkari. A Survey on Web-based AR Applications. Malaysia.2011.

[13] R. Azuma, Yohan Baillot, Reinhold Behringer, etc. Recent Advances in Augmented Reality.2001.

[14] R. Azuma et al.,A Motion-Stabilized Outdoor Augmented Reality System, Proc. IEEE Virtual Reality, IEEE CS Press, Los Alamitos, Calif, pp. 252-259. 1999. 
[15] R. Azuma, J. Leonard, Performance Analysis of an Outdoor Augmented Reality Tracking System that Relies Upon a Few Mobile Beacons, Proceedings of ISMAR 2006 (IEEE and ACM Page 17 of 30 International Symposium on Mixed and Augmented Reality), Santa Barbara, CA, Oct 22-25, 2006, pp119-122.

[16] Daniel Wagner, Tobias Langlotz, Dieter Schmalstieg. Robust and Unobtrusive Marker Tracking on Mobile Phones. Graz University of Technology.2008.

[17] Petri Honkamaa, Sanni Siltanen, Jani Jappinen. Interactive outdoor mobile augmention using markerless tracking and GPS. VTT Technical Research Centre of Finland.2007.

[18] Binghong Wang, "Research of high precision target tracking technology Based on vision in augmented reality environment," University of Electronic Science and Technology of China. 2010. (In Chinese)

[19] Xueting Tan, "Research of tracking technology of mobile augmented reality," Shanghai Jiaotong University, 2010. (In Chinese)

[20] Miaoliang Zhu, Yuan Yao, Yunliang Jiang, "Survey on Augmented Reality,” Journal of Image and Graphics, vol. 9(7), pp. 767-774, 2004. (In Chinese)

[21] Xiong Lin, et al, "Research on the key technology of mobile augmented reality system," Journal of Image and Graphics, vol. 14(3), pp. 560-564, 2009. (In Chinese)
[22] Jiang Chen, at al, "Augmented reality tracking algorithm based on feature recognition," SCIENCECHINA, vol. 40(11), pp. 1437-1449, 2010. (In Chinese)

[23] Yong Chang, "Research on Key Technologies of augmented reality of intelligent mobile phone," Computer Engineering and Applications, vol. 47(15), pp. 69-71, 2011. (In Chinese)

[24] Jiang Chen, "Application of augmented reality technology on intelligent mobile phone," Journal of University of Electronic Science and Technology of China, vol. 39, pp. 80-84, 2010. (In Chinese)

[25] Yuan Sun, Jing Chen, "Research of mobile augmented reality on mobile phones," Computer Science, vol. 39(6A), 2012. (In Chinese)

[26] Huarui $\mathrm{Wu}$, "Research on Integrated Architecture for Intelligent Agriculture Application," Computer Engineering and Design, vol. 24(9), pp. 4-7, 2003. (In Chinese)

[27] Lianwei Li, Zhenjie Wang, "Design and implementation of digital agriculture spatial information management platform,"Journal Shandong University of Technology, vol. 22(6), pp. 1-5, 2008. (In Chinese)

[28] Dujuan Zhang, Anqi Yang, Zhenfang Shan, "Research on Using the Wireless Network to Set up Agricultural," Journal of Agricultural Mechanization Research, vol. 7, pp. 19-21, 2006. (In Chinese) 